The unfortunate conclusion to be drawn is that African cooperative movements are various stages of decline rather than growth. "Il n'est personne aujourd'hui pour douter que le mouvement coopératif sénégalais ne soit gravement malade", was said of the movement in Senegal, but it applies equally today to all other African countries.

E.K.M. Yakpo

\author{
K. Ferdinand / M. Mozaffari \\ Islam: State and Society \\ Hrsg. vom Scandinavian Institute of Asian Studies \\ Curzon Press Ltd., London 1988, 219 S.
}

Das Buch enthält die Beiträge zu einem Kongreß, der im Spätsommer 1984 an der Universität Aarhus zu dem Thema "Islam - Staat und Gesellschaft" stattfand. Es gibt die unterschiedlichsten Einblicke in das Staatswesen der islamischen Welt und zeigt u.a. die gesellschaftspolitischen Probleme auf, die diesen Staaten heute bei der Bewältigung, Umsetzung oder auch Loslösung von der Maxime "la din - la dawla" (ohne Religion gibt es kein Gemeinwesen) erwachsen.

Teil I befaßt sich mit zeitgenössischen Islamstudien. Während Chr. Toll Ziel und Zweck derartiger Studien erörtert und auch einen historischen Rückblick auf die europäische Orientforschung nicht ausläßt, gibt der anschließende Beitrag von J. Hjärpe die - weitaus aktuellere - Debatte in der islamischen Welt über die in der UN-Deklaration von 1948 verankerten Menschenrechte wieder. Hjärpe schildert deutlich, warum Akzeptanz und Ablehnung der UN-Deklaration oft dicht beieinander liegen und bis heute nicht einmal über den Begriff der Menschenrechte Einigkeit erzielt werden konnte: Die UN-Deklaration als von Menschen geschaffenes Recht muß im islamischen Verständnis zwangsläufig gegenüber der Sari'a als göttlichem und alles beherrschendem Recht mängelbehaftet, lückenhaft und generell minderwertig sein mit der Folge, daß Menschenrechte, deren Verständnis im Gegensatz zur Sari'a steht, nicht als solche gelten. Nur folgerichtig war der - in Europa wenig beachtete - Entwurf einer Menschenrechtsdeklaration auf der 14. Islamischen Konferenz in Dhaka 1983, deren wichtigste Punkte Hjärpe skizziert. Kerimov befaßt sich mit islamischer Missionstätigkeit und schildert die Rolle islamischer Zentren in Europa als Kultur-Mittler, auf die insbesondere die islamische Minderheit in der Diaspora angewiesen sei. Der von manchen dieser Zentren ausgehende Mißbrauch durch Förderung politischer Aktivitäten an der Grenze der Legalität wird totgeschwiegen bis auf einen Nebensatz "carrying out various political activities". Auch sonst begegnet der Beitrag von Kerimov erheblichen inhaltlichen Bedenken - oder sollte man angesichts der Beispiele im Sudan und Iran emstlich annehmen, daß durch die Verbreitung von Sari'a-Gerichten die Zahl der 
Gewaltverbrechen abnimmt? Oder Zweifler an der christlichen Lehre zum Islam überlaufen und aus diesem Grunde bewußt eine Kenntnisnahme vom wahren Inhalt des Islams durch die Medien verhindert wird? Zu kurz und ungenau ist auch Kerimovs Feststellung, die islamische Missionierung mache Propaganda für eine Islamische Staatslehre. Was ist unter einer "Islamic Theory of State" zu verstehen? Benötigt ein islamisches Gemeinwesen zur Rechtfertigung seiner Machtausübung eine Staats- und Verfassungslehre? Genügt nicht der Qur'an? Kerimov gibt unkritisch die Standpunkte der Leiter islamischer Missionszentren wieder und überläßt dem Leser die nähere Auseinandersetzung.

Teil II des Buches ist der Staatsmacht gewidmet und vereint Beiträge über die Ursprünge des islamischen Staates, den Begriff der Staatsmacht im klassischen Gedankengut und zu Staat und Politik in der Philosophie von Shah Waliy Allah mit z.T. wörtlichen Zitaten.

Im dritten Teil des Buches werden kasuistisch die Säkularisierungsbestrebungen in einzelnen Ländern unter Einbezug ihrer geistigen Väter (al-Afghani, Abduh, Kemal Atatürk) behandelt. Am Beispiel Syriens wird gezeigt, wie sich die Opposition zur Ba'ath-Partei in der Muslimbruderschaft sammelt und der Staat - unter dem Anspruch, als integrierende Kraft zwischen den verschiedenen Klassen der Gesellschaft zu wirken - in jüngster Vergangenheit vermehrt ein vom islamischen Erbe geprägtes Vokabular benutzt, um seine Machtinteressen zu wahren. Das heißt, religiöse Symbole bedeuten hier keine Hinwendung zum islamischen Staat, sondern dienen als Fassade dem Versuch, religiöse Kreise der Bevölkerung in den weltlichen Staat zu integrieren.- Olsen erörtert die politische Bedeutung des Islams in Ägypten und Saudi-Arabien und geht dabei von der These aus, daß der Islam in der Dritten Welt keine Verändenungen bewirkt hat, sondem nur benutzt wird, um die an der Macht befindlichen politischen und wirtschaftlichen Kräfte zu legitimieren. Als Beispiel führt er die Wirtschaftspolitik unter Sadat und die Öfnung zum Kapitalismus an und weist für Saudi-Arabien darauf hin, daß die 'Ulama aus vielen ihrer ureigenen Gebiete, z.B. der Erziehung, zurückgedrängt und vom Staat angestellt und bezahlt werden.- Zwei Beiträge sind Afghanistan gewidmet; interessant ist im Hinblick auf die Entwicklung eines modernen Staates der Beitrag von Christensen mit seinen Modellzeichnungen zu den Wechselwirkungen zwischen religiöser und weltlicher Macht. Allah, König und - schließlich - Parlament werden in den verschiedenen politischen Entwicklungsstadien teils in offenen, teils in geschlossenen Kreisläufen zueinander in Beziehung gesetzt und vermitteln dem Leser so deutlich die Entwicklung zur heutigen Situation in Afghanistan, wo Allah aus der Konzeption des Staatsaufbaus eliminiert ist und damit die Idee "kein Staat ohne Islam", wie sie bis zur Regentschaft Amanullahs herrschte, aufgegeben wurde.- Der Beitrag von Naur über Gewerkschaften in Libyen gibt Einblick in die Interpretationsversuche Ghaddafi's, die Kompatibilität dieser Organisationen mit dem Islam darzustellen, und führt zur Begründung über weite Passagen das "Green Book" des Staatschefs an. Realistisch ist der Ausblick der Verfasserin, ob die von Staat und Gewerkschaft unisono propagierte Einkommensgleichheit die in der Bevölkerung aufkommende und mit dem rückgängigen Ölgeschäft zunehmende Unruhe stoppen kann.- Minderheitenprobleme insbesondere der Berber in Algerien haben ihren historischen Hintergrund in der durch die französische Kolonial- 
macht bedingten Trennung der Bevölkerung in Berberophone und Arabophone; der von Frankreich befreite, selbständige Staat verdrängte die seinerzeit schnell französisierten Berber, schwieg ihre Existenz - wegen Kollaboration mit dem Feind - quasi tot und fühlte sich erst seit der Protestbewegung der kabylischen Berber im Frühjahr 1980 veranlaßt, diese Politik aufzugeben: Seit 1983 gibt es an der Universität Algier - wieder - einen Lehrstuhl für Berbersprache und -kultur. Inzwischen wird die Berberfrage in Marokko, Mali, Niger und Frankreich diskutiert.

Insgesamt weisen die Beiträge dieses Buches auf interessante Problemstellungen, bringen aber - bedingt durch ihre Konzipierung als Vorträge - nicht stets die notwendige Breite und Tiefe. Der zur Zeit des Kongresses noch unentschiedene Problemfall Iran und die sich hier im Zusammenhang mit der Schaffung einer Theokratie aufdrängenden Fragen sind - leider ausgespart.

Dagmar Hohberger

\section{Eva Dietz}

Der Funktionswandel der Koka in Bolivien

Spektrum (Berliner Reihe zu Gesellschaft, Wirtschaft und Politik in Entwicklungsländem) Nr. 26, Verlag Breitenbach Publishers, Saarbrücken, Fort Lauderdale 1990, 138 S., DM 21,--

Kokain ist die derzeit am weitesten verbreitete und am heftigsten diskutierte Droge. Nach ihrer raschen Verbreitung in den USA drängt sie derzeit mit Macht auf den europäischen Markt. Kokain und seine Bekämpfung gehören heute weltweit zu den vorrangigen Problemen.

Zusammen mit dem Kokain ist auch Bolivien, das neben Peru wichtigste Erzeugerland des Kokablatts (des Rohstoffs für die Kokainherstellung) in die Schlagzeilen geraten. Während die Wurzeln des traditionellen Kokakonsums im Andenraum vermutlich weit vor unsere Zeitrechnung zurückreichen, ist der Anbau des Kokablatts im großen Stil zur Rohstoffgewinnung für die Kokainproduktion kaum 30 Jahre alt. Die Beschreibung dieses Funktionswandels ist das Anliegen der vorliegenden soziologischen Diplomarbeit. Die Autorin kommt zu dem richtigen Ergebnis, daß dem traditionellen Gebrauch der Koka nach wie vor eine wichtige Funktion für die indianische Mehrheit der bolivianischen Bevölkerung zukommt: Kokablätter werden von den indianischen Bauem und Bergarbeitem vornehmlich als physische und psychische Hilfsmittel benutzt. Das Kauen der Koka hat eine hunger-, durst- und schmerzstillende Wirkung. Zugleich wirkt es als Aufputschmittel. Die Koka erleichtert somit das Leben unter extremen Klima-, Höhen- (und Arbeits-!) verhältnissen. 